PROCEEDINGS OF THE

AMERICAN MATHEMATICAL SOCIETY

Volume 129, Number 12, Pages 3479-3484

S 0002-9939(01)06125-1

Article electronically published on April 25, 2001

\title{
FINITE GROUPS AND THE FIXED POINTS OF COPRIME AUTOMORPHISMS
}

\author{
PAVEL SHUMYATSKY
}

(Communicated by Stephen D. Smith)

\begin{abstract}
Let $p$ be a prime, and let $G$ be a finite $p^{\prime}$-group acted on by an elementary abelian $p$-group $A$. The following results are proved:

1. If $|A| \geq p^{3}$ and $C_{G}(a)$ is nilpotent of class at most $c$ for any $a \in A^{\# \text {, }}$ then the group $G$ is nilpotent of $\{c, p\}$-bounded class.

2. If $|A| \geq p^{4}$ and $C_{G}(a)^{\prime}$ is nilpotent of class at most $c$ for any $a \in A^{\# \text {, }}$ then the derived group $G^{\prime}$ is nilpotent of $\{c, p\}$-bounded class.
\end{abstract}

\section{INTRODUCTION}

Let $G$ be a group admitting an action of a group $A$. We denote by $C_{G}(A)$ the set $C_{G}(A)=\left\{x \in G \mid x^{a}=x\right.$ for any $\left.a \in A\right\}$, the centralizer of $A$ in $G$ (the fixedpoint group). Throughout this paper we assume that $A$ is a noncyclic elementary abelian $p$-group, and $G$ is a finite $p^{\prime}$-group. Let $A^{\#}$ denote the set of non-identity elements of $A$. It follows from the classification of finite simple groups that if $C_{G}(a)$ is solvable for any $a \in A^{\#}$, then so is the group $G$ (see [3]). The case $|A| \geq p^{3}$ does not require the classification: the result follows from Glauberman's theorem on solvable signalizer functors [1]. In certain specific situations much more can be said about the structure of $G$.

Ward showed that if $A$ has rank at least 3, and if $C_{G}(a)$ is nilpotent for any $a \in A^{\#}$, then the group $G$ is nilpotent [7. Another of Ward's results is that if $A$ has rank at least 4 , and if $C_{G}(a)^{\prime}$ is nilpotent for any $a \in A^{\#}$, then the derived group $G^{\prime}$ is nilpotent $[8$. Later the author found that if, under these assumptions, $C_{G}(a)$ is nilpotent of class at most $c$ (respectively $C_{G}(a)^{\prime}$ is nilpotent of class at most $c$ ) for any $a \in A^{\#}$, and if $G$ has derived length $d$, then the nilpotency class of $G$ (respectively of $G^{\prime}$ ) is $\{c, d, p\}$-bounded [6]. In the present paper we show that actually much stronger results are valid: the bounds on the class of $G$ and $G^{\prime}$ can be chosen independent of $d$.

Theorem 1.1. Let $A$ be an elementary abelian group of order $p^{3}$ acting on a finite $p^{\prime}$-group $G$. Assume that $C_{G}(a)$ is nilpotent of class at most $c$ for any $a \in A^{\#}$. Then $G$ is nilpotent and the class of $G$ is bounded by a function depending only on $p$ and $c$.

Received by the editors April 26, 2000.

1991 Mathematics Subject Classification. Primary 20D45.

Key words and phrases. Automorphisms, centralizers, associated Lie rings.

The author was supported by CNPq-Brazil. 
Theorem 1.2. Let $A$ be an elementary abelian group of order $p^{4}$ acting on a finite $p^{\prime}$-group $G$. Assume $C_{L}(a)^{\prime}$ is nilpotent of class at most $c$ for any $a \in A^{\#}$. Then $G^{\prime}$ is nilpotent and the class of $G^{\prime}$ is bounded by a function depending only on $p$ and $c$.

We conjecture that these results can be generalized in the following way.

Conjecture 1.3. Let $A$ be an elementary abelian group of order $p^{k}$ with $k \geq 3$ acting on a finite $p^{\prime}$-group $G$.

1. If $\gamma_{k-2}\left(C_{G}(a)\right)$ is nilpotent of class at most $c$ for any $a \in A^{\#}$, then $\gamma_{k-2}(G)$ is nilpotent and has $\{c, k, p\}$-bounded class.

2. If, for some integer $d$ such that $2^{d}+2 \leq k$, the dth derived group of $C_{G}(a)$ is nilpotent of class at most $c$ for any $a \in A^{\#}$, then the dth derived group $G^{(d)}$ is nilpotent and has $\{c, k, p\}$-bounded class.

Our main evidence in favor of the above conjecture is Lie-theoretic: Theorem 2.7 obtained in Section 2 establishes the corresponding results for Lie algebras.

\section{Action on Lie algebras}

Throughout the paper the term Lie algebra means Lie algebra over an associative ring with unity. Let $L$ be a Lie algebra. If $X, Y, X_{1}, \ldots, X_{s}$ are subsets of $L$ we use $[X, Y]$ to denote the subspace of $L$ spanned by the set $\{[x, y] \mid x \in X, y \in Y\}$. If $t \geq 2$ we write $\left[X,{ }_{t} Y\right]$ for $\left[\left[X,{ }_{t-1} Y\right], Y\right]$ and $\left[X_{1}, \ldots, X_{t}\right]$ for $\left[\left[X_{1}, \ldots, X_{t-1}\right], X_{t}\right]$. For any positive integer $w$, define commutator-spaces of weight $w$ in $X_{1}, \ldots, X_{s}$ :

A subspace of $L$ is a commutator-space of weight 1 in $X_{1}, \ldots, X_{s}$ if and only if it is the linear span of $X_{i}$ for some $i \leq s$. A subspace $M$ of $L$ is a commutator-space of weight $w \geq 2$ in $X_{1}, \ldots, X_{s}$ if and only if $M=\left[M_{1}, M_{2}\right]$, where $M_{1}$ and $M_{2}$ are commutator-spaces of weights $w_{1}$ and $w_{2}$ respectively, such that $w_{1}+w_{2}=w$.

A well-known theorem of Kreknin [5] says that if a Lie ring $L$ admits a fixedpoint-free automorphism of finite order $n$, then $L$ is solvable and the derived length of $L$ is bounded by a function of $n$. We will require the following extension of this result 4 .

Theorem 2.1. Let a Lie ring $L$ admit an automorphism a of finite order $n$ such that $\left[L,{ }_{t} C_{L}(a)\right]=0$. Assume that $n L=L$. Then $L$ is solvable with derived length at most $(t+1)^{n-1}+\log _{2} t$.

Lemma 2.2. Let $t \geq 1$. Let $L$ be a Lie algebra, and $K$ a nilpotent subalgebra of class c. Assume $K$ is generated by subspaces $X_{1}, \ldots, X_{m}$ such that for any commutator-space $Y$ in $X_{1}, \ldots, X_{m}$ we have $\left[L,{ }_{t} Y\right]=0$. Then there exists a $\{c, m, t\}$-bounded number $u$ such that $\left[L,{ }_{u} K\right]=0$.

Proof. This is by induction on $c$. Since $K^{\prime}$ is generated by commutator-spaces of weight $\geq 2$ in $X_{1}, \ldots, X_{m}$ and since the number of such spaces is $\{c, m\}$-bounded, the inductive hypothesis will be that there exists a $\{c, m, t\}$-bounded number $u_{1}$ such that $\left[L,{ }_{u_{1}} K^{\prime}\right]=0$. Now put $r=m(t-1)+1$ and consider the space $M=$ $\left[L, Y_{1}, \ldots, Y_{r}\right]$ for some choice of $Y_{1}, \ldots, Y_{r} \in\left\{X_{1}, \ldots, X_{m}\right\}$. Obviously, for any permutation $\pi$ of the symbols $1,2, \ldots, r$ we have $M \leq\left[L, Y_{\pi(1)}, \ldots, Y_{\pi(r)}\right]+\left[L, K^{\prime}\right]$. The number $r$ is big enough to ensure that some $X_{i}$ occurs in the list $Y_{1}, \ldots, Y_{r}$ at least $t$ times. Thus, we obtain $M \leq\left[L,{ }_{t} X_{i}, * \ldots, *\right]+\left[L, K^{\prime}\right]$, where the asterisks denote some spaces $Y_{j}$ which, in view of the fact that $\left[L,{ }_{t} X_{i}\right]=0$, are of no consequence. Hence, $M \leq\left[L, K^{\prime}\right]$. 
Now take $u=u_{1} r$. Using the fact that $K=K^{\prime}+\sum X_{j}$ and $M \leq\left[L, K^{\prime}\right]$ for any choice of $Y_{1}, \ldots, Y_{r} \in\left\{X_{1}, \ldots, X_{m}\right\}$, it is easy to see that $\left[L,{ }_{u} K\right] \leq\left[L, u_{1} K^{\prime}\right]=$ 0 .

Hypothesis 2.3. Let $\omega$ be a primitive pth root of unity, and let $L$ be a Lie algebra over $\mathbb{Z}[\omega]$ such that $L=p L$. Let $A$ be an elementary abelian group of order $p^{k}$ acting by automorphisms on $L$. Let $\hat{A}$ be the character group of $A$. For any $\alpha \in \hat{A}$ we set $L_{\alpha}=\left\{x \in K \mid x^{a}=\alpha(a) x\right.$ for each $\left.a \in A\right\}$.

It is well-known that $A$ and $\hat{A}$ are isomorphic, $\left[L_{\alpha}, L_{\beta}\right] \leq L_{\alpha \beta}$ for all $\alpha, \beta \in \hat{A}$ and $L=\bigoplus_{\alpha} L_{\alpha}$. For any positive integer $n$ and any $\alpha_{1}, \ldots, \alpha_{2^{n}} \in \hat{A}$ define inductively

$$
\begin{gathered}
\gamma\left(\alpha_{1}\right)=L_{\alpha_{1}} \text { and } \gamma\left(\alpha_{1}, \ldots, \alpha_{n}\right)=\left[\gamma\left(\alpha_{1}, \ldots, \alpha_{n-1}\right), L_{\alpha_{n}}\right] \\
\delta\left(\alpha_{1}\right)=L_{\alpha_{1}} \text { and } \delta\left(\alpha_{1}, \ldots, \alpha_{2^{n}}\right)=\left[\delta\left(\alpha_{1}, \ldots, \alpha_{2^{n-1}}\right), \delta\left(\alpha_{2^{n-1}+1}, \ldots, \alpha_{2^{n}}\right)\right] .
\end{gathered}
$$

As usual, $\gamma_{n}(L)$ and $L^{(n)}$ denote the $n$th term of the lower central series and the $n$th term of the derived series of $L$, respectively.

Lemma 2.4. Under Hypothesis 2.3 we have $\gamma_{n}(L)=\sum \gamma\left(\alpha_{1}, \ldots, \alpha_{n}\right)$ and $L^{(n)}=$ $\sum \delta\left(\alpha_{1}, \ldots, \alpha_{2^{n}}\right)$, where $\alpha_{1}, \ldots, \alpha_{2^{n}}$ range independently through $\hat{A}$.

Proof. Set $Q=\sum \gamma\left(\alpha_{1}, \ldots, \alpha_{n}\right)$. For any $\beta \in \hat{A}$ we have

$$
\left[\gamma\left(\alpha_{1}, \ldots, \alpha_{n}\right), L_{\beta}\right] \leq \gamma\left(\alpha_{1} \alpha_{2}, \alpha_{3}, \ldots, \alpha_{n}, \beta\right) \leq Q
$$

which shows that $Q$ is normalized by $L_{\beta}$ and therefore is an ideal of $L$. It is easy to see that $L / Q$ is nilpotent of class at most $n-1$ and so $\gamma_{n}(L) \leq Q$. The opposite inclusion is obvious, whence $\gamma_{n}(L)=Q$.

To prove the other claim we set $R_{n}=\sum \delta\left(\alpha_{1}, \ldots, \alpha_{2^{n}}\right)$ and, arguing by induction on $n$, assume that $R_{n-1}=L^{(n-1)}$. We now need to show that $R_{n}=R_{n-1}^{\prime}$.

For any $\beta_{1}, \ldots, \beta_{2^{n-1}} \in \hat{A}$ we see that

$$
\left[\delta\left(\alpha_{1}, \ldots, \alpha_{2^{n}}\right), \delta\left(\beta_{1}, \ldots, \beta_{2^{n-1}}\right)\right] \leq R_{n}
$$

which shows that $\delta\left(\beta_{1}, \ldots, \beta_{2^{n-1}}\right)$ normalizes $R_{n}$. Therefore $R_{n}$ is an ideal in $R_{n-1}$ and it follows that $R_{n}=R_{n-1}^{\prime}$.

Corollary 2.5. Assume Hypothesis [2.3. Then, for any $\beta \in \hat{A}$, we have $L_{\beta} \cap$ $\gamma_{n}(L)=\sum \gamma\left(\alpha_{1}, \ldots, \alpha_{n}\right)$, where the summation is taken over those $\alpha_{1}, \ldots, \alpha_{n} \in \hat{A}$ for which $\alpha_{1} \ldots \alpha_{n}=\beta$. Similarly, $L_{\beta} \cap L^{(n)}=\sum \delta\left(\alpha_{1}, \ldots, \alpha_{2^{n}}\right)$, where $\alpha_{1} \ldots \alpha_{2^{n}}$ $=\beta$.

Lemma 2.6. Assume Hypothesis 2.3 with $k \geq 2$. Suppose there exists an integer $u$ such that $\left[L,{ }_{u} C_{L}(a)\right]=0$ for any $a \in A^{\#}$. Then $L$ is nilpotent of $\{p, u\}$-bounded class.

Proof. By Theorem 2.1] $L$ is solvable and the derived length $d$ of $L$ is at most $(u+1)^{p-1}+\log _{2} u$. We will prove the lemma by induction on $d$. Applying the inductive hypothesis to $L^{\prime}$ assume that $L^{\prime}$ is nilpotent of $\{p, u\}$-bounded class $e$, say. 
Let $B$ be any subgroup of $A$ of order $p^{2}$, and let $B_{1}, \ldots, B_{p+1}$ be the cyclic subgroups of $B$. We set $C_{i}=C_{L}\left(B_{i}\right), 1 \leq i \leq p+1$. Then $L=\sum_{i} C_{i}$. Let $r=(u-1)(p+1)+1$. If $Z=Z\left(L^{\prime}\right)$ we obviously have $[Z, X, Y]=[Z, Y, X]$ for any subsets $X, Y$ of $L$. Having this in mind we write

$$
\left[Z,{ }_{r} L\right]=\left[Z,{ }_{r} \sum_{i} C_{i}\right]=\sum\left[Z,{ }_{u_{1}} C_{1}, \ldots, u_{p+1} C_{p+1}\right],
$$

where $u_{1}+u_{2}+\ldots u_{p+1}=r$. The number $r$ is big enough to ensure that $u_{i} \geq u$ for some $i$, so it follows that $\left[Z,{ }_{u_{1}} C_{1}, \ldots, u_{p+1} C_{p+1}\right]=0$ since $\left[L,{ }_{u} C_{i}\right]=0$. Thus, $\left[Z,{ }_{r} L\right]=0$ and therefore $Z \leq Z_{r}(L)$, where $Z_{r}(L)$ is the $r$ th term of the upper central series of $L$. Applying this argument repeatedly to $L / Z, L / Z_{2}\left(K^{\prime}\right)$ and so on, we conclude that $L^{\prime} \leq Z_{e r}(L)$ and therefore $L$ is of nilpotency class at most er +1 .

Theorem 2.7. Assume Hypothesis 2.3 with $k \geq 3$.

1. If $\gamma_{k-2}\left(C_{L}(a)\right)$ is nilpotent of class at most $c$ for any $a \in A^{\#}$, then $\gamma_{k-2}(L)$ is nilpotent and has $\{c, k, p\}$-bounded class.

2. If, for some integer $d$ such that $2^{d}+2 \leq k$, the dth derived group of $C_{L}(a)$ is nilpotent of class at most $c$ for any $a \in A^{\#}$, then $L^{(d)}$ is nilpotent and has $\{c, k, p\}$-bounded class.

Proof. 1. Obviously, for any $\beta, \alpha_{1}, \ldots, \alpha_{k-2} \in \hat{A}$ there exists $a \in A^{\#}$ such that $L_{\beta}, L_{\alpha_{1}}, \ldots, L_{\alpha_{k-2}} \leq C_{L}(a)$. Since $\gamma_{k-2}\left(C_{L}(a)\right)$ is nilpotent of class at most $c$, it follows that $\left[L_{\beta}, c+2 \gamma\left(\alpha_{1}, \ldots, \alpha_{k-2}\right)\right]=0$. Now, using that $L=\bigoplus_{\beta} L_{\beta}$, we derive that $\left[L, c+2 \gamma\left(\alpha_{1}, \ldots, \alpha_{k-2}\right)\right]=0$. Corollary 2.5 shows that $C_{L}(a) \cap \gamma_{k-2}(L)=$ $\sum \gamma\left(\alpha_{1}, \ldots, \alpha_{k-2}\right)$, where the summation is taken over all those $\alpha_{1}, \ldots, \alpha_{k-2}$ for which $\alpha_{1} \ldots \alpha_{k-2}(a)=1$. We now apply Lemma 2.2 with $K=C_{L}(a) \cap \gamma_{k-2}(L)$ and the spaces $\gamma\left(\alpha_{1}, \ldots, \alpha_{k-2}\right) \leq C_{L}(a)$ in place of $X_{i}$ to deduce that there exists a $\{c, k, p\}$-bounded number $u$ such that $\left[L,{ }_{u} K\right]=0$. But then it follows from Lemma [2.6 that $\gamma_{k-2}(L)$ is nilpotent of $\{p, u\}$-bounded class.

2. The proof of the second claim is not really much different from what we have done above. We establish first that $\left[L,{ }_{c+2} \delta\left(\alpha_{1}, \ldots, \alpha_{2^{d}}\right)\right]=0$ for all $\alpha_{1}, \ldots, \alpha_{2^{d}} \in$ $\hat{A}$. Next, we apply Lemma 2.2 to deduce that there exists a $\{c, k, p\}$-bounded number $u$ such that $\left[L,{ }_{u} C_{L^{(d)}}(a)\right]=0$ for all $a \in A$. Finally, we observe that the required assertion follows from Lemma 2.6

\section{MAIn RESULTS}

The next lemma is well-known (see [2, 6.2.2, 6.2.4] for the proof).

Lemma 3.1. Let $A$ be a finite $p$-group acting on a finite $p^{\prime}$-group $G$.

1. If $N$ is an $A$-invariant normal subgroup of $G$, then $C_{G / N}(A)=C_{G}(A) N / N$.

2. If $A$ is an elementary abelian group, and if $A_{1}, \ldots, A_{s}$ are the maximal subgroups of $A$, then $G=\left\langle C_{G}\left(A_{i}\right) \mid 1 \leq i \leq s\right\rangle$.

Lemma 3.2. Let $p$ be a prime, and $G$ a finite $p^{\prime}$-group acted on by an elementary abelian p-group $A$ of rank at least 3 . Let $A_{1}, \ldots, A_{s}$ be the maximal subgroups of A. Then

$$
G^{\prime}=\left\langle\left[C_{G}\left(A_{i}\right), C_{G}\left(A_{j}\right)\right] \mid 1 \leq i, j \leq s\right\rangle .
$$


Proof. By Lemma $3.1 G=\left\langle C_{G}\left(A_{1}\right), \ldots, C_{G}\left(A_{s}\right)\right\rangle$. Consider the subgroup $R=$ $\left\langle\left[C_{G}\left(A_{i}\right), C_{G}\left(A_{j}\right)\right] \mid 1 \leq i, j \leq s\right\rangle$. Obviously $R$ is $A$-invariant so $R=\left\langle C_{R}\left(A_{1}\right), \ldots\right.$, $\left.C_{R}\left(A_{s}\right)\right\rangle$. To show that $R$ is normal it is sufficient to establish that $y^{x} \in R$ for any $y \in C_{R}\left(A_{i}\right)$ and $x \in C_{G}\left(A_{j}\right)$. We have $y^{x}=y^{x} y^{-1} y$ and obviously both $y^{x} y^{-1}$ and $y$ belong to $R$. Hence $y^{x} \in R$ and $R$ is normal. Using that $G=$ $\left\langle C_{G}\left(A_{1}\right), \ldots, C_{G}\left(A_{s}\right)\right\rangle$, it is now easy to see that $G / R$ is abelian, as required.

We are now ready to prove the main results.

Theorem 1.1. Let $A$ be an elementary abelian group of order $p^{3}$ acting on a finite $p^{\prime}$-group $G$. Assume that $C_{G}(a)$ is nilpotent of class at most $c$ for any $a \in A^{\#}$. Then $G$ is nilpotent and the class of $G$ is bounded by a function depending only on $p$ and $c$.

Proof. We know from Ward's result cited in the Introduction that $G$ is nilpotent. Let $L(G)$ be the Lie ring corresponding to the lower central series of $G$. The construction associating the Lie ring with $G$ is well-known. Let $\gamma_{i}$ denote the $i$ th term of the lower central series of $G$. Set $L_{i}=\gamma_{i} / \gamma_{i+1}$ and view $L_{i}$ as an additive abelian group. Then $L(G)=\bigoplus_{i} L_{i}$. If $x \in \gamma_{i}, y \in \gamma_{j}$, then, for corresponding elements $x \gamma_{i+1}, y \gamma_{j+1}$ of $L(G)$, we set $\left[x \gamma_{i+1}, y \gamma_{j+1}\right]=[x, y] \gamma_{i+j+1}$. This operation can be uniquely extended by linearity on the additive abelian group $L(G)$ and, equipped with the product, $L(G)$ becomes a Lie ring. The Lie ring has the same nilpotency class as $G$. In our situation the group $A$ acts naturally on each quotient $\gamma_{i} / \gamma_{i+1}$ and this action extends uniquely to an action by automorphisms on the Lie ring $L(G)$. Lemma 3.1 shows that if $a \in A$, then $C_{L(G)}(a)$ is the direct sum of the quotients $C_{\gamma_{i}}(a) \gamma_{i+1} / \gamma_{i+1}$. It follows that $C_{L(G)}(a)$ is nilpotent of class at most $c$ for any $a \in A^{\#}$. Finally, we note that $L(G)$ is finite and has the same order as $G$. Therefore $p L(G)=L(G)$. Set $L=L(G) \otimes \mathbb{Z}[\omega]$. We can view $L$ as a Lie algebra over $\mathbb{Z}[\omega]$ and $A$ as a group acting on $L$. By Theorem $2.7 L$ is nilpotent of $\{c, p\}$-bounded class and so is $G$.

Theorem 1.2. Let $A$ be an elementary abelian group of order $p^{4}$ acting on a finite $p^{\prime}$-group $G$. Assume $C_{L}(a)^{\prime}$ is nilpotent of class at most $c$ for any $a \in A^{\#}$. Then $G^{\prime}$ is nilpotent and the class of $G^{\prime}$ is bounded by a function depending only on $p$ and $c$.

Proof. Let $A_{1}, \ldots, A_{s}$ be the maximal subgroups of $A$. Then, by Lemma 3.2, $G^{\prime}=$ $\left\langle\left[C_{G}\left(A_{i}\right), C_{G}\left(A_{j}\right)\right] \mid 1 \leq i, j \leq s\right\rangle$. We know that $G^{\prime}$ is nilpotent. Let $L\left(G^{\prime}\right)$ be the Lie ring corresponding to the lower central series of $G^{\prime}$. Set $L=L\left(G^{\prime}\right) \otimes \mathbb{Z}[\omega]$. We will view $L$ as a Lie algebra over $\mathbb{Z}[\omega]$ and $A$ as a group acting on $L$. By Theorem 2.7 $L^{\prime}$ is nilpotent of $\{c, p\}$-bounded class, say $e$. Let $X_{1}, \ldots, X_{t}$ be the images of various subgroups of the form $\left[C_{G}\left(A_{i}\right), C_{G}\left(A_{j}\right)\right]$ in $G^{\prime} / G^{\prime \prime}$. So $L$ is generated by the sets $X_{1}, \ldots, X_{t}$. For any $i, j, k \leq s$ we observe that there exists some $a \in A^{\#}$ such that the centralizers $C_{G}\left(A_{i}\right), C_{G}\left(A_{j}\right), C_{G}\left(A_{k}\right)$ are all contained in $C_{G}(a)$. Therefore $\left[C_{G}\left(A_{k}\right),{ }_{c+2}\left[C_{G}\left(A_{i}\right), C_{G}\left(A_{j}\right)\right]\right]=1$. Now, if $X_{l}$ is the image of $\left[C_{G}\left(A_{i}\right), C_{G}\left(A_{j}\right)\right]$ in $G^{\prime} / G^{\prime \prime}$, it follows that $\left[C_{L}\left(A_{k}\right),{ }_{c+2} X_{l}\right]=0$, whence $\left[L,{ }_{c+2} X_{l}\right]=0$.

Set $r=(c+1) t+1$. If $Z=Z\left(L^{\prime}\right)$, we obviously have $[Z, X, Y]=[Z, Y, X]$ for any subsets $X, Y$ of $L$. Having this in mind, and taking into account that $L$ is generated by the sets $X_{i}$, we write

$$
\left[Z,{ }_{r} L\right]=\sum\left[Z,{ }_{u_{1}} X_{1}, \ldots, u_{t} X_{t}\right]
$$


where $u_{1}+u_{2}+\cdots+u_{t}=r$. The number $r$ is big enough to ensure that $u_{j} \geq c+2$ for some $j$, so it follows that $\left[Z,{ }_{u_{1}} X_{1}, \ldots, u_{t} X_{t}\right]=0$ since $\left[L,{ }_{c+2} X_{j}\right]=0$. Thus, $\left[Z,{ }_{r} L\right]=0$ and therefore $Z \leq Z_{r}(L)$, where $Z_{r}(L)$ is the $r$ th term of the upper central series of $L$. Applying this argument repeatedly to $L / Z, L / Z_{2}\left(K^{\prime}\right)$ and so on, we conclude that $L^{\prime} \leq Z_{e r}(L)$ and therefore $L$ is of nilpotency class at most $e r+1$.

\section{REFERENCES}

[1] G. Glauberman, On solvable signalizer functors in finite groups, Proc. London Math. Soc. 33 (1976), 1-27. MR 54:5341

[2] D. Gorenstein, Finite groups, New York, Evanston, London: Harper and Row, 1968. MR 38:229

[3] R. Guralnick and P. Shumyatsky, Derived subgroups of fixed points, preprint, 2000.

[4] E. I. Khukhro and P. Shumyatsky, On fixed points of automorphisms of Lie rings and locally finite groups, Algebra and Logic 34 (1995), 395-405. MR 97c:20059

[5] V.A. Kreknin, Solvability of Lie algebras with a regular automorphism of finite period, Soviet Math. Dokl. 4 (1963), 683-685.

[6] P. Shumyatsky, On periodic soluble groups and the fixed point groups of operators, Comm. Algebra 20(10) (1992), 2815-2820. MR 93i:20038

[7] J.N. Ward, On finite groups admitting automorphisms with nilpotent fixed-point group, Bull. Austral. Math. Soc. 5 (1971), 281-282. MR 45:5224]

[8] J.N. Ward, On finite soluble groups and the fixed-point groups of automorphisms, Bull. Austral. Math. Soc. 5 (1971), 375-378. MR 45:3572

Department of Mathematics, University of Brasilia, Brasilia-DF, 70910-900 Brazil

E-mail address: pavel@ipe.mat.unb.br 